RESPIRATORY INFECTION

\title{
Seasonality of long term wheezing following respiratory syncytial virus lower respiratory tract infection
}

\author{
L Bont, M Steijn, W M C van Aalderen, F Brus, J M Th Draaisma, R A A M Van \\ Diemen-Steenvoorde, M Pekelharing-Berghuis, J L L Kimpen
}

Thorax 2004;59:512-516. doi: 10.1136/thx.2003.013391

See end of article for authors' affiliations

Correspondence to:

Correspondence to:
Dr J L L Kimpen,

Department of Pediatric

Infectious Diseases,

Wilhelmina Children's

Hospital, University

Medical Center, 3508 AB

Utrecht, The Netherlands:

i.kimpen@wkz.azu.nl

Received 22 July 2003

Accepted

23 February 2004

\begin{abstract}
Background: It is well known that respiratory syncytial virus (RSV) lower respiratory tract infection (LRTI) is associated with subsequent wheezing episodes, but the precise natural course of wheezing following RSV LRTI is not known. This study aimed to determine the continuous development of wheezing following RSV LRTI in children up to the age of 3 years.

Methods: A prospective cohort study was performed in 140 hospitalised infants with RSV LRTI. Continuous follow up data were obtained with a unique log in which parents noted daily respiratory symptoms.

Results: A marked decrease in wheezing was seen during the first year of follow up. The burden of wheezing following RSV LRTI was observed during the winter season. Signs of airflow limitation during RSV LRTI were strongly associated with wheezing during the follow up period. Total and specific serum immunoglobulin E levels, patient eczema, and parental history of atopy were not associated with wheezing.

Conclusions: Airway morbidity following RSV LRTI has a seasonal pattern, which suggests that viral upper respiratory tract infections are the predominant trigger for wheezing following RSV LRTI. There is a significant decrease in airway symptoms during the first 12 months after admission to hospital. Simple clinical variables, but not allergic risk factors, can predict the development of wheezing following RSV LRTI.
\end{abstract}

$\mathrm{R}$ ecurrent episodes of wheezing in early childhood following respiratory syncytial virus (RSV) bronchiolitis have well been documented and occur in $42-71 \%$ of cases. ${ }^{1}$ It is thought that these wheezing episodes are triggered by viral upper respiratory tract infections (URTI). ${ }^{2}$ Wheezing following RSV lower respiratory tract infections (LRTI) appears to be independent of atopy, ${ }^{3}$ although an association has been found with allergy. ${ }^{24-8}$ Follow up studies have shown bronchial hyperresponsiveness $4-8$ years after admission to hospital with RSV bronchiolitis. ${ }^{28} 9$ Although it is clear that RSV LRTI is associated with subsequent wheezing, no continuous data on respiratory symptoms and long term airway morbidity following RSV LRTI are available. Infants who go on to have wheezing episodes do so within 12-24 months after the first episode of RSV LRTI. ${ }^{2}$ Data in the literature show that wheezing following RSV LRTI is transient. ${ }^{3}$ The Tucson birth cohort study shows that wheezing at age 6 years is no longer associated with a history of RSV LRTI before the age of 3 years. ${ }^{8}$ More precise information on the development of wheezing following RSV LRTI is, however, unavailable.

Questionnaires have been used to estimate the relation between RSV LRTI and subsequent wheezing. ${ }^{56810}$ It is difficult to assess how accurate a questionnaire is in providing data on airway symptoms following RSV LRTI. In particular, recall bias by parents might interfere with accuracy. In addition, lung function measurements have been performed to address pulmonary function following RSV LRTI, ${ }^{8}{ }^{11} 12$ but it is not known how well lung function relates to respiratory symptoms. ${ }^{13}$ Moreover, lung function is measured from the age of 6 years while airway symptoms have already substantially decreased by that time.

To overcome the limitations of currently used follow up methods, we performed a prospective long term log based study to demonstrate the continuous development over time of airway morbidity following RSV LRTI. We hypothesised that the severity of wheezing following RSV LRTI decreases over 3 years after admission to hospital. In addition, we hypothesised that wheezing following RSV LRTI has a seasonal pattern.

\section{METHODS}

\section{Study population}

One hundred and forty children at six hospitals in the Netherlands were prospectively studied during two winter epidemics. Two hospitals had a paediatric intensive care unit. Inclusion criteria were hospital admission, lower respiratory tract symptoms, age $<13$ months, and positive immunofluorescence for RSV in epithelial cells from nasopharyngeal secretions. There were no standard criteria used for admission to hospital. Symptoms of LRTI ${ }^{8}$ were severe chest cough, wheezing, hoarseness, stridor, shortness of breath, cyanosis and apnoea. Infants with wheezing respiration before RSV infection were excluded. A history of premature birth and the presence of physical sounds of airflow limitation on admission (expiratory wheeze or prolonged expiration) were recorded during hospitalisation. The medical ethical committee approved the study in all participating centres. Parents of subjects gave written informed consent.

\section{$\log$}

Starting 3 weeks after discharge from hospital, parents recorded respiratory symptoms, airway medication, and doctors' visits on a daily basis in a log until the age of 3 years (fig 1). Parents were instructed how to use the log by one of the investigators (LB). Daily respiratory symptoms recorded included "runny nose", "coughing", and "wheezing". The absence of respiratory symptoms was also noted. To improve compliance, telephone contact was maintained every 3 months during the entire follow up period. The completed $\log$ was returned to the investigators by mail every 3 months. Respiratory symptoms were analysed per quarter. Since infants were not all hospitalised on the same date, the 
number of wheezing days for the first quarter was corrected according to the number of missing days during the first quarter. To quantify respiratory symptoms following RSV LRTI, the numbers of days of wheezing were counted and classified as $0,1-5$, or $>5$ wheezing days/quarter. The main outcome variable was the percentage of children with $>5$ wheezing days/quarter. In addition, the number of wheezing episodes experienced by each child was counted. ${ }^{14} \mathrm{~A}$ disease episode was defined as the presence of respiratory symptoms for two or more consecutive days. Children were considered lost to follow up if more than two logs (two quarters) were not returned.

\section{Questionnaire}

At the end of the follow up period (age 3 years) an extended Dutch version of the standardised questionnaire of the British Medical Council and the Dutch version of the European Community Respiratory Health Survey questionnaire were used to obtain data on respiratory symptoms, allergy, parental smoking habits, medication, and allergy symptoms among first degree family members. ${ }^{15}{ }^{16}$ Parental history of atopy was defined as the presence of asthma, eczema, or hay fever in at least one parent.

\section{Serum immunoglobulin E}

At the end of the follow up period (age 3 years) total IgE and specific IgE against a panel of common inhalation (house dust mite Dermatophagoides farinae, cat dander, dog dander, mixed grass pollen) and food allergens (cow's milk, egg white, soya bean, wheat, peanut) were measured in serum by
CAP system FEIA (Pharmacia Diagnostics, Uppsala, Sweden). The geometric mean (SD) of a large normal control population measured at our laboratory at age 3 years is 8.0 (32) $\mathrm{kU} / \mathrm{l}$.

\section{Statistical analysis}

The numbers of days and episodes of wheezing are expressed as median values. Serum IgE is expressed as geometric mean. Because there is no gold standard for atopic status, four atopic parameters were distinguished: patient eczema, parental history of atopy, total serum IgE, and allergen specific IgE. Differences in sex, prematurity, requirement of mechanical ventilation, parental history of atopy between infants with and without signs of airflow limitation during the initial RSV LRTI were analysed using a $\chi^{2}$ test. Differences in age, number of episodes of wheezing or coughing, and number of days of coughing or wheezing between infants with and without signs of airflow limitation during the initial RSV LRTI were analysed using the MannWhitney U test.

Imputation of data for missing logs was performed to allow for statistical analysis of incomplete data. Missing values were replaced with the mean of the previous and subsequent values. When values at the end of the third follow up year were missing, these missing values were replaced by the mean of the previous value and the value of the same quarter during the previous year. Thus, missing values were replaced by values that were completely dependent from observations of the same child. To estimate the potential effect of imputation on the model, we compared our model with the

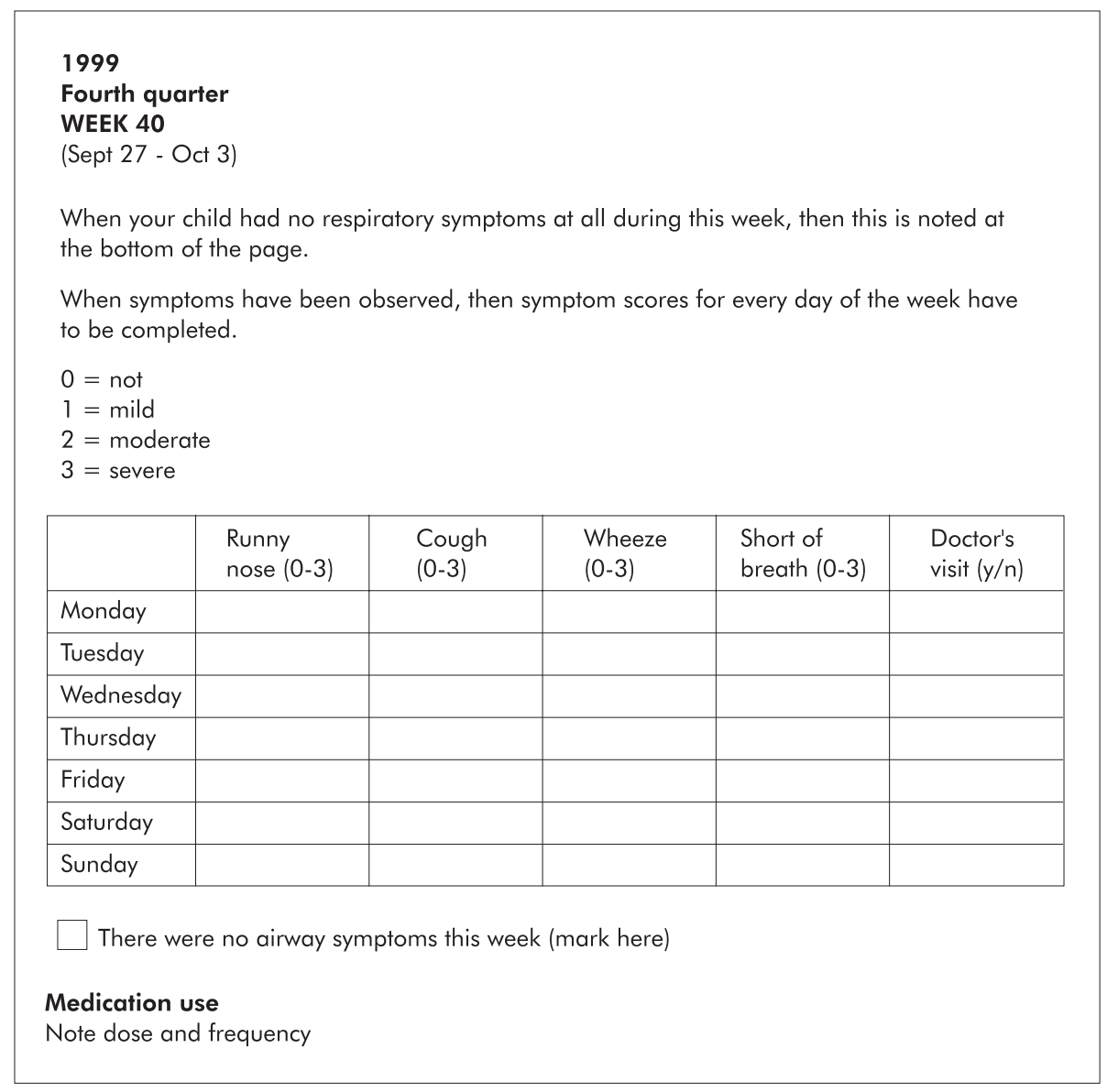

Figure 1 Example of a log page (English translation). One log page is used to note daily respiratory symptoms during 1 week. The presence or absence of "runny nose", "cough", and "wheeze" were noted. Severity of symptoms and presence or absence of respiratory distress were not analysed. 
model that would result from an alternative approach for imputation. Using this alternative imputation strategy, missing values were imputed by the mean of non-missing values of other children during the same quarter of the same follow up year. Thus, with the alternative approach missing values were replaced by values that were completely independent of observations from the same child. Following imputation, analysis of log derived follow up data was performed using general linear model (GLM) repeated measures analysis. We analysed the simple predictive value of relevant parameters for recurrent wheezing, as well as the predictive value in complex models with more than one predictor. Post hoc Bonferroni correction was performed. All statistics was performed with Statistical Package for the Social Sciences (SPSS), version 10.0. Significance was defined by a two sided $\alpha$ level of 0.05 .

\section{RESULTS}

\section{Subject characteristics}

One hundred and forty children (77 boys) of median age 2 months with RSV LRTI were enrolled (table 1). Forty one patients $(29 \%)$ were born prematurely (median 34 weeks, range 25-36 weeks). Twenty nine subjects $(21 \%)$ needed mechanical ventilation. Four patients $(3 \%)$ had cardiac disease and two $(2 \%)$ had chronic lung disease. Signs of airflow limitation were noted in $84(60 \%)$. None of the patients received ribavirin or systemic corticosteroids during their initial RSV LRTI. All patients survived RSV LRTI.

\section{Wheezing following RSV LRTI}

Complete follow up data were available for 106 children; a single log was missing from 19 children, two logs were missing from seven children, and eight children were lost during the follow up period, of whom one had died 3 months after RSV LRTI from meningococcal disease. Wheezing days and episodes per quarter are shown in figs 2 and 3. The results were compared using two different imputation models. Differences between both models were limited and therefore we conclude that imputation of missing values had little effect on our results.

Wheezing following RSV LRTI was characterised by two novel findings. Firstly, a sharp decrease of $>50 \%$ in wheezing was observed during the first year of follow up $(p<0.001)$. In particular, the percentage of children with severe wheezing ( $>5$ days/quarter) decreased. Secondly, an increase in respiratory wheeze occurred during the winter periods in all three study years $(\mathrm{p}<0.001)$.

The clinical follow up data in the logs and questionnaires were compared (table 2). An underestimation of "ever wheeze" was found in the questionnaires compared with the log registrations, particularly for children who only wheezed during the first year of follow up. Another major finding which was not anticipated was the overestimation of

\begin{tabular}{|c|c|}
\hline Variable & \\
\hline $\begin{array}{l}\text { Boys } \\
\text { Premature } \\
\text { Median (range) age (weeks) } \\
\text { Exclusively breast fed during infancy } \\
\text { On mechanical ventilation during RSV } \\
\text { Signs of airflow limitation during RSV LRTI } \\
\text { Parental history of atopy } \\
\text { Smoke exposure before or after RSV LRTI }\end{array}$ & $\begin{array}{l}77(55 \%) \\
41(29 \%) \\
7(0-52) \\
42(30 \%) \\
29(21 \%) \\
84(60 \%) \\
62(44 \%) \\
29(21 \%)\end{array}$ \\
\hline
\end{tabular}

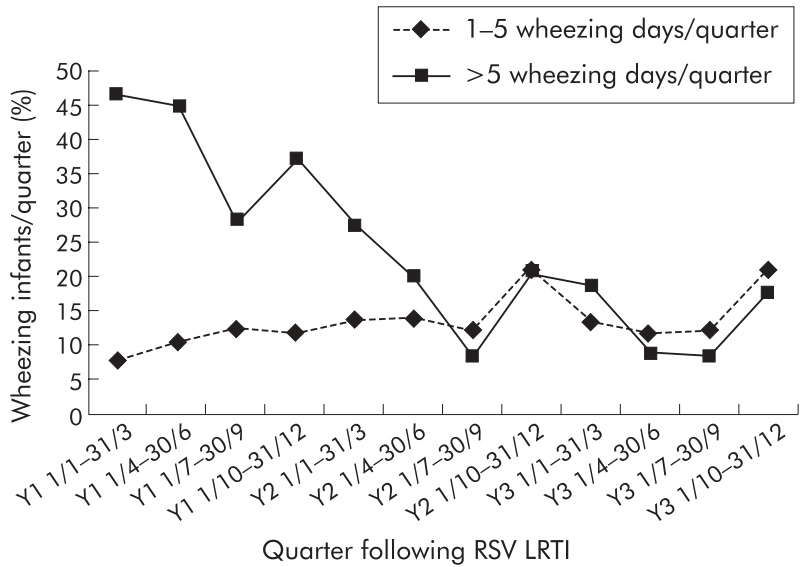

Figure 2 Number of days with respiratory wheeze per quarter following RSV LRTI. Parents recorded daily respiratory symptoms which were analysed per quarter. Infrequent wheeze (1-5 days/quarter) and frequent wheeze ( $>5$ days/quarter) are distinguished.

respiratory wheeze by parents during the previous 12 months in the questionnaires compared with the log registrations.

We analysed the predictive value of relevant clinical parameters for wheezing following RSV LRTI. Sex, age at onset of RSV LRTI, mechanical ventilation, or neonatal breast feeding were not related to wheezing during the follow up period. In addition, we did not find a relation between environmental tobacco smoke exposure before or after inclusion in the study and wheezing during follow up. Signs of airflow limitation during RSV LRTI (expiratory wheeze or prolonged expiration) were significantly associated with wheezing during the follow up period $(p<0.001$, fig 4$)$. Baseline characteristics of infants with and without signs of airflow limitation during RSV LRTI were similar (data not shown).

\section{Atopic parameters in relation to wheeze during follow up}

We analysed the association between patient eczema or parental history of atopy and wheezing during the follow up period. No significant association was found between eczema or parental history of atopy and the total number of episodes/ days of wheezing during the whole follow up period and for

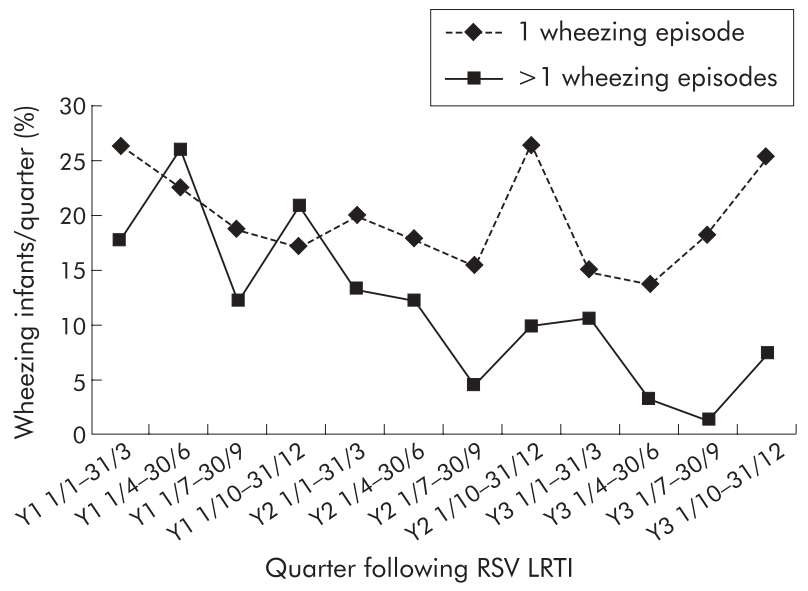

Figure 3 Number of episodes of respiratory wheeze per quarter following RSV LRTI. An episode is defined as respiratory symptoms for more than 1 day. Infants with 1 and $>1$ wheezing episodes per quarter are distinguished. 
Table 2 Comparison between log derived and questionnaire derived follow up data

\begin{tabular}{lllll}
\hline & & & Discordance \\
\cline { 3 - 4 } & Questionnaire & Log & Quest+/log- & Quest-/log+ \\
\hline $\begin{array}{l}\text { Ever wheezing } \\
\text { Wheezing in }\end{array}$ & $79(60 \%)$ & $110(83 \%)$ & $1(1 \%)$ & $32(24 \%)$ \\
previous 12 months & $56(42 \%)$ & $47(36 \%)$ & $23(17 \%)$ & $14(11 \%)$ \\
\hline & & & \\
\hline
\end{tabular}

each follow up year separately (not shown). Serum IgE was determined in the 126 infants who completed the study. In six infants no blood was obtained. Mean serum IgE was 11 (95\% CI 9-15) kU/l. Infants were defined as having high IgE $(>25 \mathrm{kU} / \mathrm{l}$, highest quarter) or low $\operatorname{IgE}(\leqslant 25 \mathrm{kU} / \mathrm{l})$. The association between total or allergen specific IgE and wheezing during the follow up period was analysed (not shown). No significant association was found between total or allergen specific IgE and the total number of episodes/days of wheezing during the whole follow up period or for each follow up year separately. We tested whether atopic parameters were predictive of wheezing during follow up when analysed together in one GLM, but no significant relation was found.

To evaluate whether serum IgE or parental history were appropriate indicators of atopic status we analysed the association between total and allergen specific serum IgE, parental history of atopy, and eczema. Patient eczema was associated with parental history of atopy $(\mathrm{p}<0.01)$ but not with total or allergen specific serum IgE. Parental history of atopy appeared to be an appropriate indicator of atopy in infants but was not related to airway morbidity following RSV LRTI.

\section{DISCUSSION}

To our knowledge, this is the first long term log based longitudinal study in a large cohort of infants admitted to hospital with RSV LRTI. Recurrent wheezing markedly decreased during the first 3 years following hospitalisation with RSV LRTI. An increase in wheezing during the winter season has not previously been reported. Signs of airflow limitation during RSV LRTI were predictive of recurrent

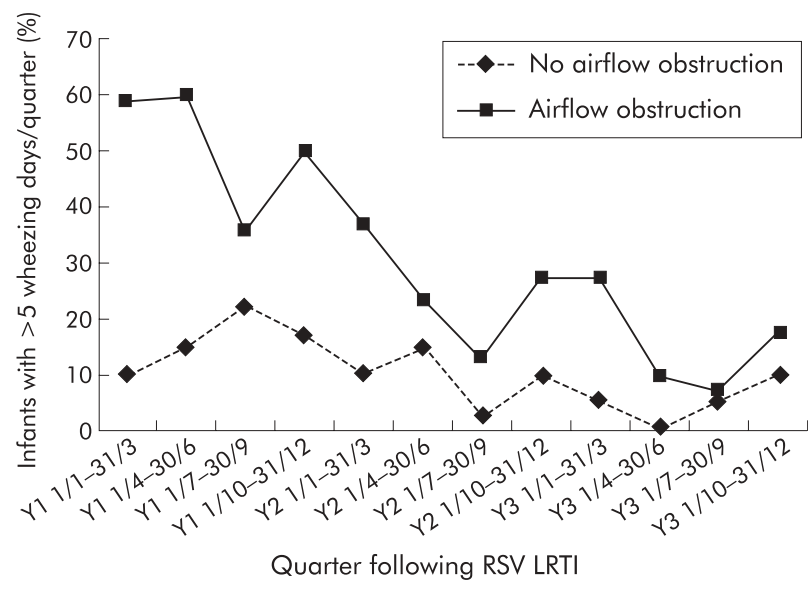

Figure 4 Days of respiratory wheeze following RSV LRTI in relation to airflow obstruction during RSV LRTI. Parents recorded daily respiratory symptoms which were analysed per quarter. The proportion of infants with frequent wheeze ( $>5$ days/quarter) are shown. Patients with and without signs of airflow limitation are distinguished $(p<0.001)$. wheeze during follow up. Atopic characteristics such as parental history of atopy, specific IgE, or patient eczema were not related to recurrent wheeze following RSV LRTI.

Previous studies have shown that wheezing following RSV LRTI is transient. ${ }^{358}$ It has been reported that wheezing following RSV LRTI usually subsides by the age of 4-6 years. The rapid fall in the number of wheezing days during the first year of follow up in this study has not previously been reported. It is unlikely that this decrease in airway symptoms reflects decreased adherence to the log for two reasons: firstly, fig l shows that parents were instructed to fill out the log even when there were no symptoms, and secondly, the seasonal increase in reported symptoms suggests adequate adherence. Previous studies have used repeated questionnaires to measure airway morbidity following RSV LRTI. In these studies questionnaires are used for the first time 13 years after the RSV LRTI. These studies have therefore been unable to demonstrate a strong continuous decrease in wheezing during the first year of follow up.

The seasonal pattern of wheezing following RSV LRTI may provide a clue to triggers of wheezing episodes. Wheezing episodes following RSV LRTI are thought to be the result of increased exposure to respiratory viruses during the autumn and winter seasons. ${ }^{2}$ This would be in agreement with a community based longitudinal study (not focusing on RSV) which found that viral URTI occur during wheezing episodes in asthmatic school children. ${ }^{17}$ The seasonal increase in the prevalence of viral infections in autumn and winter coincides with the seasonal increase in house dust mite exposure ${ }^{18}$ and, consequently, bronchial hyperresponsiveness. We cannot therefore exclude the possibility that differences in allergen exposure contribute to the seasonality of wheezing following RSV LRTI. ${ }^{19}$

The results of this study may be of direct clinical use. Although indications for admission to hospital may not be uniform throughout the world, infants admitted with RSV LRTI form a recognisable group of patients for paediatricians. To date, it is difficult to estimate expected long term airway morbidity following RSV LRTI for individual infants. An increased risk of recurrent wheeze exists until school age, which is probably independent of atopic factors. ${ }^{2}{ }^{8}$ This study provides three additional clinically useful tools to estimate more precisely long term airway morbidity following RSV LRTI - the marked decrease in wheezing during the first year of follow up following RSV LRTI, the seasonal pattern, and the predictive value of signs of airflow limitation during RSV LRTI for subsequent wheeze.

The relationship between RSV LRTI and the development of allergy is intriguing. Sigurs et al showed an increased risk for allergy in hospitalised patients with RSV at ages 3 and 7 years compared with matched controls, ${ }^{4}{ }^{20}$ but other studies have not been in agreement with these findings. ${ }^{2} 37101221$ Most convincingly, Stein and colleagues showed in a birth cohort study that RSV LRTI before the age of 4 years is not associated with subsequent allergic sensitisation, irrespective of disease severity. ${ }^{8}$ Our study was not designed to analyse 
the association between RSV LRTI and allergy. However, our data indicate a limited role for atopy in the pathogenesis of wheezing following RSV LRTI. The absence of a relationship between a family history of atopy, patient eczema, or serum IgE at age 3 years on the one hand and wheezing following RSV LRTI on the other hand shows that (the larger part of) wheezing following RSV LRTI cannot be explained by atopy. Thus, in line with previous studies, we conclude that wheezing following RSV LRTI is not caused by allergy induction. $^{3}$

We compared the respiratory symptoms derived from questionnaires with log derived data and found a discrepancy in reported wheezing. As expected, some parents noted wheezing in the log but did not confirm this in the questionnaire. This could be explained by parents forgetting symptoms (recall bias). We had not anticipated that a substantial proportion of parents would mention in the questionnaire that their child had had wheezing during the past 12 months but would not note this in the log. This too could be explained by recall bias, with parents forgetting that the last episode of wheezing occurred more than 12 months ago. We did not attempt to validate log data. However, in the absence of a gold standard, log derived data may provide an accurate tool for analysing airway morbidity following RSV LRTI.

This log based longitudinal study is the first report on long term daily respiratory symptoms in infants admitted to hospital with RSV LRTI. The results show that airway morbidity following RSV LRTI markedly decreases during the first year after hospitalisation. The seasonal pattern of wheezing following RSV LRTI is not only of direct clinical use, but also suggests that viral URTI are the predominant trigger for wheezing following RSV LRTI. No relationship was found between wheezing following RSV LRTI and atopy. Clinical variables during RSV LRTI such as signs of airflow limitation can be used to predict subsequent wheezing.

\section{ACKNOWLEDGEMENTS}

The authors thank N Kortenhoef and B Tonies (University Medical Center, Utrecht, The Netherlands) for assistance with collection of questionnaire data, H Hartman for excellent assistance with statistical analysis, and the Dutch Asthma Foundation for funding the work (grant 96.08).

\section{Authors' affiliations}

L Bont, M Steijn, J L L Kimpen, Wilhelmina Children's Hospital, University Medical Center, Utrecht, The Netherlands

W M C van Aalderen, Academic Medical Center, Amsterdam, The Netherlands

F Brus, Department of Pediatrics, Rijnstate Hospital, Arnhem, The Netherlands
J M Th Draaisma, Department of Pediatrics, St Elisabeth Hospital, Tilburg, The Netherlands

R A A M Van Diemen-Steenvoorde, Department of Pediatrics, St Antonius Hospital, Nieuwegein, The Netherlands

M Pekelharing-Berghuis, Department of Pediatrics, Diakonessen Hospital, Utrecht, The Netherlands

This study was supported by the Dutch Asthma Foundation (grant 32.96.08)

\section{REFERENCES}

1 Bont L, Aalderen van WMC, Kimpen JLL. Long-term consequences of respiratory syncytial virus (RSV) bronchiolitis. Paediatr Respir Rev 2000;1:221-7.

2 Pullan CR, Hey EN. Wheezing, asthma and pulmonary dysfunction 10 years after infection with respiratory syncytial virus infection in infancy. BMJ 1982;284:1665-9.

3 Eisen AH, Bacal HL. The relationship of acute bronchiolitis to bronchial asthma at 4 to 14 year follow-up. Pediatrics 1963;31:859-61.

4 Sigurs N, Bjarnason R, Sigursbergsson F, et al. Asthma and immunoglobulin E antibodies after respiratory syncytial virus bronchiolitis: a prospective cohort study with matched controls. Pediatrics 1995;95:500-5.

5 Sims DG, Downham MA, Gardner PS. Study of 8-year-old children with a history of respiratory syncytial virus bronchiolitis in infancy. $B M J$ 1978;1:11-17

6 McConnochie KM, Roghmann KJ. Bronchiolitis as a possible cause of wheezing in childhood: new evidence. Pediatrics 1984;74:1-10.

7 Hall CB, Hall WJ, Gala CL, et al. Long-term prospective study in children after respiratory syncytial virus infection. J Pediatr 1984;105:358-64.

8 Stein RT, Sherill D, Morgan WJ, et al. Respiratory syncytial virus in early life and risk of wheeze and allergy by age 13. Lancet 1999;354:541-5.

9 Welliver RC, Duff AL. The relationship of RSV-specific immunoglobulin Eantibody responses in infancy, recurrent wheezing and pulmonary function at age 7-8 years. Pediatr Pulmonol 1993;15:19-27.

10 Sly PD, Hibbert ME. Childhood asthma following hospitalization with acute viral bronchiolitis in infancy. Pediatr Pulmonol 1989;7:153-8.

11 Dezateux C, Fletcher ME, Dundas I, et al. Infant respiratory function after RSVproven bronchiolitis. Am J Respir Crit Care Med 1997;155:1349-55.

12 Gurwitz D, Mindorff C, Levinson H. Increased evidence of bronchial reactivity in children with a history of acute bronchiolitis. J Pediatr 1981;98:551-5.

13 Marchal F, Loos N. Lung function testing in preschool children. Pediatr Pulmonol Suppl 1999;18:21-3.

14 Bont L, Heijnen CJ, Kavelaars A, et al. Monocyte IL-10 production during respiratory syncytial virus bronchiolitis is associated with recurrent wheezing in a one-year follow-up study. Am J Respir Crit Care Med 2000;161:1518-23.

15 Burney PG, Luczynska C, Chinn S, et al. The European Community Respiratory Health Survey. Eur Respir J 1994;7:954-60.

16 Rijcken B. Het Europees luchtwegonderzoek Nederland. Tijdschr Soc Gezondheidsz 1992;70:259-60.

17 Johnston SL, Pattemore PK, Sanderson G, et al. Community study of role of viral infections in exacerbations of asthma in 9-11 year old children. BMJ 1995;310:1225-8.

18 Meijer GG, Postma DS, van der Heide S, et al. Seasonal variations in house dust mite influence the circadian peak expiratory flow amplitude. Am J Respir Crit Care Med 1996;154:881-4.

19 Bielory L, Deener A. Seasonal variation in the effects of major indoor and outdoor environmental variables on asthma. J Asthma 1998;35:7-48.

20 Sigurs N, Bjarnason R, Sigurbergsson F, et al. Respiratory syncytial virus bronchiolitis in infancy is an important risk factor for asthma and allergy at age 7. Am J Respir Crit Care Med 2000;161:1501-7.

21 Murray M, Webb MSC, O'Callaghan C, et al. Respiratory status and allergy after bronchiolitis. Arch Dis Child 1992;67:482-7. 